'Departamento de Cirugía Oncológica y Maxilofacial. División de Cirugía. Hospital Clínico Pontificia Universidad Católica de Chile. Santiago, Chile. ${ }^{2}$ Sección de Medicina de Urgencia. Hospital Clínico Pontificia Universidad Católica de Chile. Santiago, Chile.

Los autores no declaran conflicto de intereses. El trabajo no recibió financiamiento.

Recibido el 23 de mayo de 2016, aceptado el 21 de octubre de 2016.

Correspondencia a: Andrés Campolo González Marcoleta\#350, Santiago, Chile. andres.campolo@gmail.com

\section{Manejo del trauma maxilofacial en la atención de urgencia por no especialistas}

\author{
ANDRÉS CAMPOLO G. ${ }^{1}$, ALLAN MIX V. ${ }^{2}$, CAMILA FONCEA R. ${ }^{1}$, \\ HERNÁN RAMÍREZ S. ${ }^{1}$, ALEX VARGAS D. ${ }^{1}$, IGNACIO GOÑI E. ${ }^{1}$
}

\section{Management of facial injuries in the emergency room}

Facial injuries are cause of consultation in emergency departments. The maxillofacial region contains several structures that are vital for life. Hence, an early assessment and management of facial injuries is important to avoid their consequential complications and eventual mortality. This article is a review of the literature about the emergency clinical assessment and management of traumatic facial injuries by non-specialists.

(Rev Med Chile 2017; 145: 1038-1046)

Key words: Emergency Medicine; Facial Bones; Life Support Care; Maxillofacial Injuries.

\footnotetext{
$\mathrm{E}$
} 1 trauma maxilofacial es una patología prevalente y compleja, siendo necesario una comprensión cabal del mecanismo y etiología, en pos de lograr un adecuado manejo.

Según la Organización Mundial de la Salud, el trauma se define como "daño intencional o no intencional causado al organismo por una brusca exposición a fuentes de energía que sobrepasan su margen de tolerancia ${ }^{1}$; mientras que el traumatismo craneofacial (TCF) se describe como aquel que afecta al complejo dentoalveolar, tejidos blandos y óseos craneofaciales. Esto puede ocurrir de manera aislada o concomitante a otras lesiones graves del organismo, especialmente con las de la región intracraneana y/o cervical ${ }^{2}$.

La etiología del TCF difiere entre países por razones culturales, sociales y factores ambientales. Las principales causas registradas en todo el mundo corresponden a caídas, accidentes de tránsito, accidentes deportivos y violencia interpersonal ${ }^{3-6}$. Esta distribución etiológica tiene principal relevancia al afectar al grupo etario joven, generando importante impacto social.

La región maxilofacial contiene estructuras esenciales para la vida; un traumatismo puede generar una amenaza vital debido a compromiso de la vía aérea, lesiones intracraneales o hemorragias, y generar importantes secuelas visuales, déficit funcional y estético a largo plazo. Es fundamental la evaluación y manejo temprano de estas patologías con el fin de reducir la morbimortalidad y evitar la necesidad de reconstrucciones complejas posteriormente ${ }^{7,8}$.

Este artículo tiene por objetivo presentar una revisión de la literatura, enfocándose en el triage, evaluación y manejo de urgencias maxilofaciales.

\section{Mortalidad del trauma}

En el año 1983, el Dr. Donald Trunkey describió que las muertes por trauma presentan una distribución trimodal. Dentro de los primeros $60 \mathrm{~min}$ se presenta el primer peak, que incluye las muertes inmediatas o muy tempranas, representando aproximadamente $45 \%$ de todas las muertes por trauma (asociadas principalmente a traumatismos cerebrales graves o del sistema cardiovascular). El 
segundo peak incluye muertes tempranas (entre $1 \mathrm{a} 4 \mathrm{~h}$ ) y representan $34 \%$; son causadas por lesiones neurológicas o cardiovasculares. El tercer peak corresponde a muertes tardías, mayor a una semana, asociadas a infecciones y fallas orgánicas, representando alrededor de $20 \%$ del total de fallecidos $^{9}$. En la actualidad existe un debate sobre este concepto trimodal clásico, puesto que la literatura más reciente ha determinado que existirían otros factores más determinantes que el tiempo ${ }^{10,11}$. Sin embargo, lo que queda claro es que la gran mayoría de las muertes prevenibles requieren de una evaluación e intervención rápida, por lo que se refuerza la importancia del manejo prehospitalario, la derivación a centros con capacidad de resolución y el concepto de la "hora de oro" para permitir una adecuada reanimación del paciente.

\section{Triage}

Pese a no existir un estándar internacional que haya demostrado superioridad en la realización de triage, el Centro de Control y Prevención de Enfermedades de Estados Unidos ha establecido guías para la realización del triage en pacientes traumatizados. De acuerdo a ella existen cuatro niveles de evaluación para establecer la gravedad de un paciente y la necesidad de derivación a un servicio de urgencia hospitalario ${ }^{11,12}$.

El primer paso corresponde a criterios fisiológicos:

- Escala de coma de Glasgow $\leq 13$.

- Presión arterial sistólica $<90 \mathrm{mmHg}$.

- Frecuencia respiratoria $<10$ o $>29$ respiraciones por minutos.

- Necesidad de soporte ventilatorio.

El segundo paso corresponde a los criterios anatómicos:

- Heridas penetrantes, mutilaciones, fracturas pélvicas o craneales.

El tercer paso es evaluar el mecanismo del trauma:

- Energía involucrada; mecanismos de alta energía son criterios de estudio.

Por último, el cuarto paso son las consideraciones especiales:

- Edad.

- Comorbilidades.

- Embarazo.

\section{Evaluación primaria y reanimación}

La evaluación primaria tiene como objetivo identificar las lesiones y condiciones del paciente, de manera sistemática, permitiendo la identificación de acciones tiempo dependiente. La idea es identificar la mayor amenaza para la vida y realizar precozmente las maniobras necesarias para reanimar al paciente. El enfoque se basa en los principios fundamentales del manejo del trauma, dentro de los que se destaca aquellos descritos por la $\mathrm{ATLS}^{13}$ :

1. $\mathrm{ABCDE}$ (protección de la vía aérea/columna cervical, ventilación, circulación/control de la hemorragia, discapacidad; estado neurológico y exposición/amenazas del medio ambiente).

2. Tratamiento de lesiones que amenazan la vida dentro de la "hora de oro".

3. Revaluación constante.

4. Considerar la importancia del mecanismo de la lesión para anticipar qué lesiones pueden estar presentes.

\section{Examen físico maxilofacial}

El examen comienza con la inspección, evaluación de asimetrías, equimosis, y hematomas, presencia de dolor, inspección del SCALP, laceraciones faciales y presencia de cuerpos extraños ${ }^{8,19}$.

Luego se realiza la palpación de los contornos de estructuras óseas buscando deformidades o diástasis. El maxilar debe ser palpado bimanualmente buscando movilidad del segmento óseo, palpar el cuerpo y arco cigomático, borde inferior mandibular y la rama. Se debe evaluar la dinámica mandibular en guías excursivas (apertura, cierre y lateralidades), buscando cualquier limitación mecánica. La palpación de los cóndilos mandibulares debe ser bilateral, en reposo y dinámica, buscando la presencia de dolor, aumentos de volumen o silencio condíleo ${ }^{8}$.

Si se sospecha una fuga de líquido cefalorraquídeo, se debe realizar una evaluación por el equipo de neurocirugía y considerar el uso de profilaxis antibiótica (Figura 1$)^{19}$.

Se sugiere realizar evaluación sensorial del paciente con ojos cerrados (nervio trigémino en sus ramos: $\mathrm{V} 1$ región frontal, $\mathrm{V} 2$ región infraorbitaria, y V3 en labio inferior y mentón). Mientras que 
las lesiones motoras del nervio facial se pueden evaluar mediante instrucciones: levantar la frente (ramo temporal), cerrar los ojos (ramo cigomático), inflar las mejillas y mostrar los dientes (ramo bucal), mover el labio inferior (ramo marginal mandibular $)^{8}$.

En el complejo nasal se evalúa asimetría y movilidad. Un espéculo nasal se utiliza para localizar áreas de sangrado o hematoma, particularmente en el tabique nasal. Un hematoma septal no reconocido puede generar necrosis del cartílago septal subyacente, por lo que debe drenarse inmediatamente ${ }^{19}$.

En caso de disminución de la agudeza visual, percepción alterada de la luz, o lesiones traumáticas del globo ocular, se debe realizar una evaluación por oftalmología para descartar cualquier compromiso severo de los globos oculares. Desde el punto de vista maxilofacial se debe evaluar el rango completo de movilidad ocular, buscando signos de atrapamiento muscular ocular ${ }^{8}$.

La evaluación intraoral se realiza buscando laceraciones de la mucosa, hematomas de piso de boca, fracturas dento-alveolares, alteraciones de la oclusión dental y descarga posterior. Ante

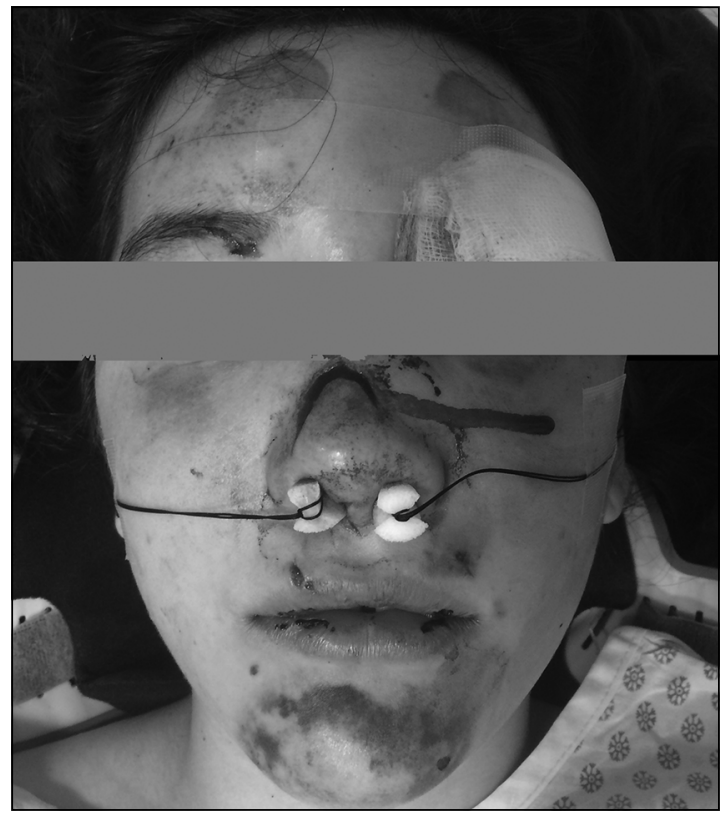

Figura 1. Fractura de tercio medio por trauma de alta energía. Ante esta signología facial, debe sospecharse fractura compleja de la cara, fractura de base de cráneo con o sin pérdida de LCR. la sospecha de un rasgo de fractura mandibular, se puede realizar una manipulación cuidadosa bimanual de los segmentos óseos buscando probar la movilidad.

Es importante destacar que el examen maxilofacial va de la mano en muchas oportunidades con la evaluación primaria. Muchas condiciones del trauma cráneofacial pueden comprometer la vía aérea, columna cervical, generar hemorragias, entre otros.

En trauma cráneofacial es importante buscar dirigídamente eventuales obstrucciones de la vía aérea en donde las causas pueden ser múltiples; hemorragias de estructuras faciales $u$ orales, aspiración de cuerpos extraños, regurgitación de contenidos gástricos, obstrucción con la lengua en pacientes inconscientes o con fracturas mandibulares bilaterales (Figura 2) ${ }^{14}$. Es por lo tanto, de importancia contar con herramientas que permitan el retiro, la aspiración y el posicionamiento de la vía aérea.

La cavidad oral debe ser aspirada si es que hay secreciones, sangre o vómito para posteriormente ser evaluada. Cualquier cuerpo extraño (prótesis dental, fragmento de fractura dentoalveolar $\mathrm{u}$ otro) debe ser removido inmediatamente por el riesgo de aspiración. Las fracturas faciales, lesiones faríngeas, laríngeas o traqueales deben ser reconocidas puesto que son potenciales causas de obstrucción por hemorragia o edema ${ }^{8,11,15}$.

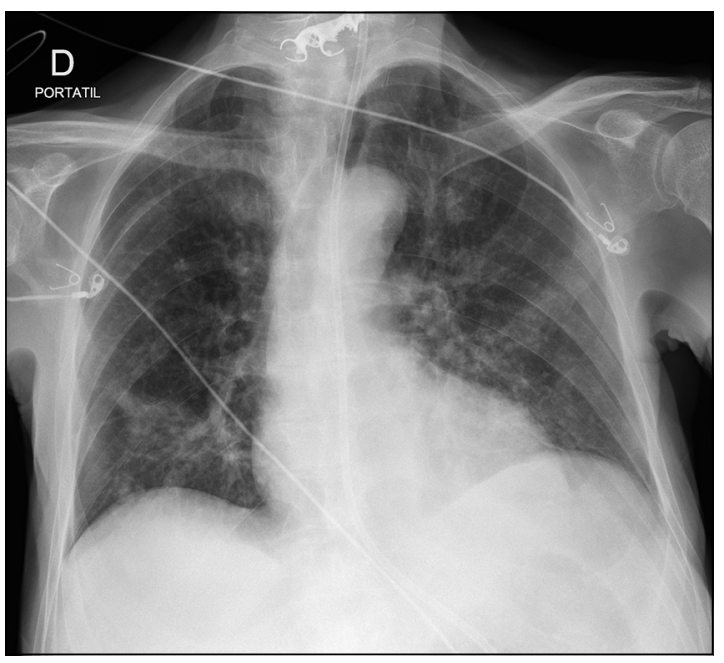

Figura 2. Radiografía de Tórax. Se observa imagen radiopaca a nivel cervical, correspondiente a prótesis dental aspirada. 
Trismus acompañado de sialorrea, babeo, estridor, disnea o hemoptisis son signos de una pérdida de la permeabilidad en la vía aérea. El trismus puede ser causado por dolor, inflamación o por compresión muscular de fragmentos óseos. Si es muy marcado ( $<25 \mathrm{~mm}$ de apertura bucal $)$ puede ser necesario asegurar la vía aérea ${ }^{8,14,16}$.

Si el paciente presenta compromiso de conciencia, se debe considerar la intubación precoz.

Las indicaciones para el manejo avanzado de vía aérea e intubación son ${ }^{11}$ :

1. Incapacidad para ventilar: Por obstrucción de las vías aéreas, trauma torácico, parálisis o fatiga respiratoria.

2. Incapacidad para oxigenar: Lesión por inhalación, síndrome de dificultad respiratoria aguda o edema pulmonar.

3. Protección de las vías aéreas respiratorias: Estado mental de inconsciencia, o conciencia alterada, traumatismo cráneofacial grave (fractura panfacial), sangrado oral abundante con riesgo de aspiración.

Se debe tener especial consideración ante la sospecha de fracturas de base de cráneo, ya que la instrumentalización nasotraqueal se encuentra contraindicada. El riesgo de realizar una inserción intracraneal lleva a un mal pronóstico en el contexto de un traumatismo craneofacial. Por lo tanto, en pacientes con fracturas de tercio medio facial (región maxilar, nasal, malar y orbitaria), no se deberán instalar sondas nasogástricas ni nasoenterales, y en caso de compromiso de la vía aérea, está contraindicado el acceso nasotraqueal, debiendo realizarse preferentemente intubación por vía orotraqueal o quirúrgico percutáneo de urgencia (Figura 3) ) $^{17,18}$.

En casos de una vía aérea difícil con obstrucción de la vía aérea alta, el manejo quirúrgico de urgencia de la vía aérea no debe retrasarse, siendo indicado la realización de una cricotiroidotomía de urgencia ${ }^{15}$.

En la literatura se describe que alrededor del 0,3 a $19,3 \%$ de los pacientes que presentan fracturas faciales, tienen algún tipo de lesión cervical asociada. Por lo tanto, además de la vía aérea, es importante evaluar la columna cervical de manera simultánea al comienzo de la evaluación del paciente ${ }^{11,19}$.

Se ha reportado que las lesiones del macizo facial están más comúnmente asociadas con trauma a nivel de $\mathrm{C} 1 / \mathrm{C} 2$ y $\mathrm{C} 6 / \mathrm{C} 7$ siendo las lesiones más frecuentes las contusiones de médula espinal y la herniación discal ${ }^{19-21}$.

El uso de collar cervical semi-rígido permite una inmovilización temporal de la columna cervical, protegiéndola de un daño mayor hasta que se realiza su estabilización. Los collares cervicales rígidos deben ajustarse correctamente, y esto es particularmente importante en la presencia de fracturas mandibulares. Se debe considerar que los collares cervicales pueden dificultar el manejo de la vía aérea, por lo que se recalca la importancia de priorizar la permeabilidad de ésta, reduciendo al mínimo los movimientos cervicales.

Se ha señalado que los collares elevan significativamente la presión intracraneal por lo que podrían ser deletéreos en casos de sospecha de lesiones cerebrales ${ }^{15,22}$. Al respecto, ante sospecha de lesiones cerebrales, se deben priorizar las medidas de neuroprotección, con cabeza fija en línea media, minimizando los movimientos rotacionales.

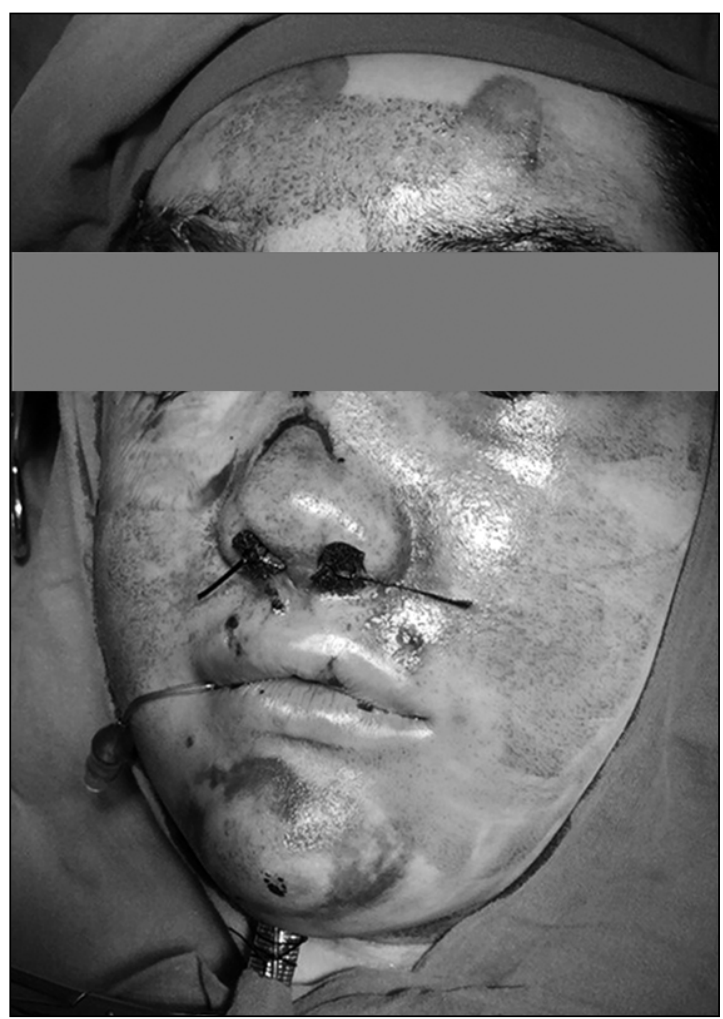

Figura 3. Intubación submentoniana en trauma facial complejo. Paciente con contraindicación para intubación nasal. 
Existen herramientas para la evaluación clínica de la columna: "National Emergency X-Radiography Utilization Study" (NEXUS) y el Canadian C-Spine Rule (CCR) son de utilidad para determinar la necesidad de estudios imagenológicos del paciente con trauma. Sin embargo, los traumatismos maxilofaciales son considerados en el grupo de alto riesgo de estos algoritmos, por lo que su aplicación es limitada ${ }^{23-25}$.

Dentro del estudio de imagenología en pacientes que no se puede realizar un buen examen clínico de la columna cervical, las opciones disponibles son: Radiografías simples en 3 planos (lateral, antero-posterior y antero-posterior transoral), tomografía computarizada y resonancia magnética ${ }^{15,22}$.

La hemorragia representa $30-40 \%$ de la mortalidad en trauma. El shock hipovolémico es una causa común de morbilidad y mortalidad. En los pacientes politraumatizados pueden haber varios sitios de pérdida de sangre, de los cuales la región maxilofacial (incluido el SCALP) puede estar involucrado. A pesar de que la hipovolemia que comprometa la vida producto de una hemorragia en la región facial es poco común, el reconocimiento y la intervención temprana son necesarias ${ }^{22,26}$.

Dada su exposición, el sangrado de la región maxilofacial puede ser alarmante en apariencia, sin embargo, raramente es responsable de hipotensión profunda. En contexto de hipotensión o shock, es labor del tratante buscar dirigídamente otros sitios de hemorragia asociados. Es relevante notar que collares cervicales pueden dificultar la identificación de laceraciones principalmente en la zona occipital ${ }^{15,22}$.

Hemorragias orofaríngeas deglutidas causan irritación gástrica y vómitos, produciendo peligro de aspiración hacia la vía aérea ${ }^{15}$.
Las laceraciones faciales y de mucosa oral pueden ser controladas por cierre con suturas y medidas hemostáticas locales. En caso de hemorragias profusas, se debe considerar la precoz evaluación por el especialista en cirugía maxilofacial, dado que algunos pacientes pueden incluso llegar a requerir de una reducción inmediata de fracturas mediante el uso de arcos dentales y bloqueo intermaxilar temporal (Figura 4) ${ }^{19}$.

La epistaxis ya sea anterior o posterior, es común después de un traumatismo maxilofacial. Si la hemorragia es profusa se puede realizar un buen taponamiento inicial usando distintos tipos de materiales (ejemplo: gasa, esponja deshidratada, hidrocoloides, etc). En algunos casos el control definitivo requiere la reducción de la fractura y el taponamiento anterior ${ }^{19,22}$.

El sangrado posterior se asocia con laceración de la arteria etmoidal posterior y por lo general se puede lograr el control mediante el uso de un catéter de globo de doble lumen (e.g. sonda vesical) de $30 \mathrm{ml}$ inflado en la nasofaringe además de un taponamiento anterior. Sin embargo, es importante considerar que el uso de catéteres puede llevar a desplazamiento de estructuras óseas fracturadas principalmente en la base del cráneo ${ }^{22,26}$.

\section{Evaluación neurológica}

Se debe realizar una evaluación íntegra del estado neurológico del paciente y reevaluar frecuentemente. Especial énfasis debe ponerse en los nervios craneales, los que deben ser evaluados según el protocolo habitual ${ }^{8,19}$.

El estudio imagenológico con tomografía computada es el estándar de calidad en traumatismo encefalocraneano ${ }^{8}$, siendo solo evitable, según las guías clínicas nacionales, en el paciente con GCS $15 \mathrm{y} \sin$ factores de riesgo (Tabla 1$)^{27}$.

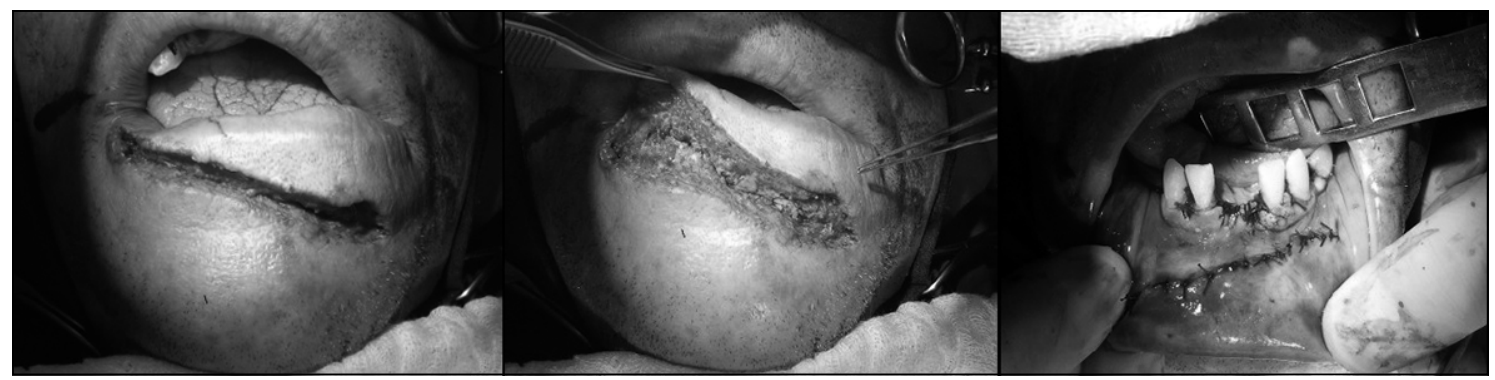

Figura 4. Herida transfixiante de labio inferior. Lesión de labio inferior que compromete plano cutáneo, muscular y mucoso, además de piezas dentarias y reborde alveolar antero-inferior. 
Urgencias de trauma maxilofacial para no especialistas - A. Campolo et al

Tabla 1. Factores de riesgo

\begin{tabular}{|c|c|}
\hline \multirow[t]{3}{*}{ Antecedentes del accidente } & Mecanismos de alta energía \\
\hline & Muerte de uno de los accidentados \\
\hline & Sospecha de lesión penetrante de cráneo \\
\hline \multirow[t]{7}{*}{ Antecedentes del paciente } & Edad $>65$ años \\
\hline & Epilepsia \\
\hline & Tratamiento anticoagulante oral o coagulopatía previa \\
\hline & Antecedentes de enfermedad neuroquirúrgica previa \\
\hline & Alcoholismo crónico \\
\hline & Abuso de drogas \\
\hline & Paciente sin apoyo social \\
\hline \multirow[t]{6}{*}{ Elementos de la anamnesis } & Segunda consulta \\
\hline & Pérdida de conciencia mayor a 5 min \\
\hline & Cefalea intensiva y progresiva \\
\hline & Vómitos explosivos \\
\hline & Presencia de convulsiones \\
\hline & Amnesia pre o post traumática \\
\hline \multirow[t]{5}{*}{ Hallazgos del examen físico y neurológico } & Presencia de déficit neurológico \\
\hline & Presencia de otorragia \\
\hline & Presencia de otorragia y rinorraquia \\
\hline & Signos de fractura de base de cráneo (ojos de mapache, signo de Battle) \\
\hline & Agitación psicomotora \\
\hline Estudio radiológico & Presencia de fractura de cráneo \\
\hline
\end{tabular}

*Extraído de Guías Clínicas del Traumatismo encéfalo-craneano, de MINSAL (2007).

Es común encontrar durante el examen neurológico la presencia de parestesias en fracturas del tercio medio facial o en fracturas mandibulares, las cuales están asociadas principalmente a la compresión de los ramos del nervio trigémino. La parálisis de ramos del nervio facial puede presentarse en casos de heridas penetrantes o laceraciones en el trayecto del nervio facial ${ }^{28}$. La mayoría de estas lesiones tienen resolución espontánea, por lo que su manejo por especialidad se debe realizar diferidamente.

\section{Evaluación oftalmológica}

La incidencia de lesiones oculares asociadas a lesiones maxilofaciales varía en la literatura del 1 a $70 \%$ y se presenta con más frecuencia en fracturas de huesos frontales ${ }^{19}$. Por lo tanto, una vez descartadas las amenazas a la vida del paciente, la atención de urgencia de un paciente con trauma craneofacial o maxilofacial debe incluir una evaluación oftalmológica.

La derivación oportuna al oftalmólogo es clave para evitar secuelas graves, $y$ las indicaciones para derivación son: cualquier lesión penetrante del globo ocular, cuerpo extraño intraocular, rotura del globo, reducción de agudeza o campo visual, lesiones corneales, hifema, deformidad de la pupila, reflejo fotomotor alterado, hemorragia vítrea, desprendimiento de retina ${ }^{19,29}$.

La evaluación oftalmológica debe incluir un examen de la córnea, conjuntiva, párpados, evaluación de campo y agudeza visual, movimientos oculares y reflejo pupilares. Las posiciones relativas del canto medial y lateral deben ser evaluadas en búsqueda de desprendimientos del ligamento cantal o una lesión naso-orbito-etmoidal ${ }^{22}$.

Otro aspecto relevante es la presencia de hematoma retrobulbar, el cual puede llevar a un 


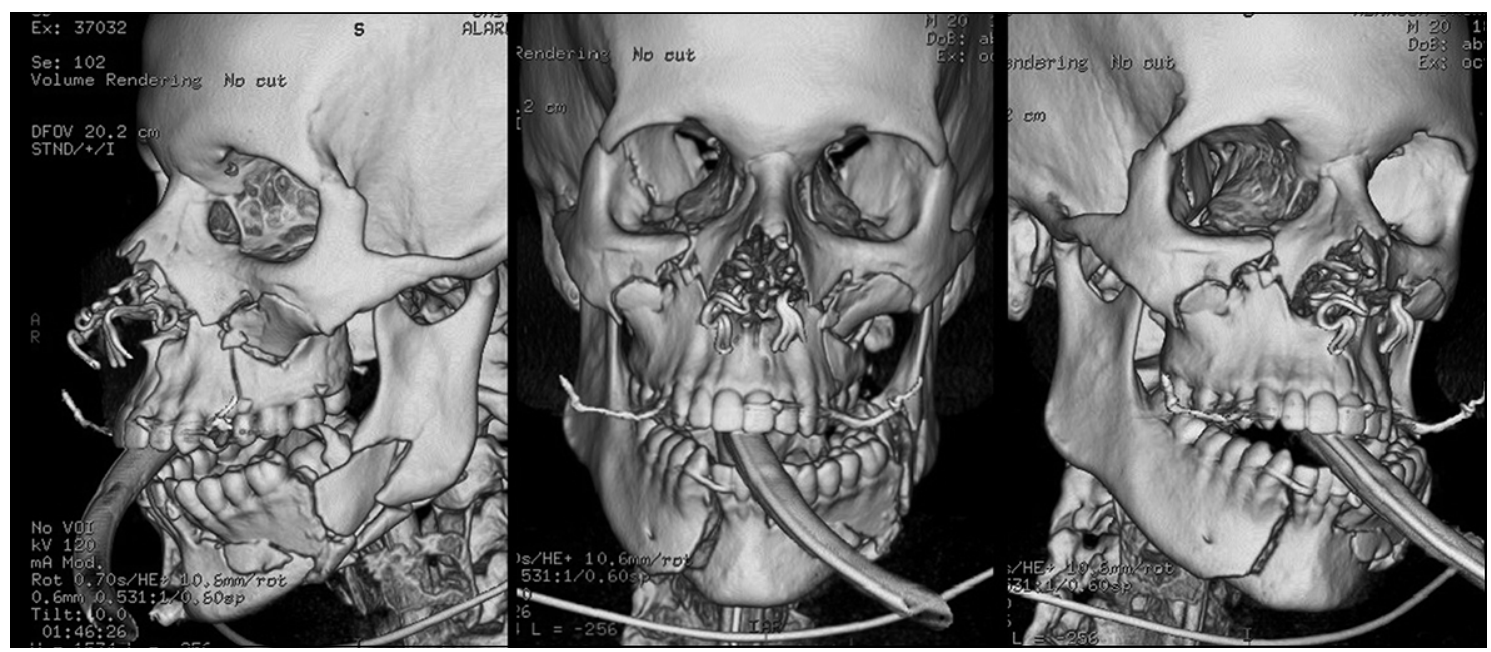

Figura 5. TC, Reconstrucción tridimensional de fractura panfacial Le Fort III derecha, Le Fort I izquierda, y fractura de cuerpo mandibular bilateral.

aumento de la presión intraorbitaria, compresión del nervio óptico y amaurosis. Los signos incluyen dolor, percepción visual reducida, pérdida del reflejo pupilar directo, preservación del reflejo consensual, proptosis y oftalmoplejia ${ }^{8}$.

Ante la sospecha de un hematoma retrobulbar, está indicada la realización de una cantotomía lateral.

\section{Imagenología}

El estudio radiológico tiene como limitante la sobreproyección de las estructuras presentes en la región maxilofacial.

En la actualidad se considera a la tomografía computarizada del macizo facial como el examen de elección y gold standard para evidenciar y caracterizar rasgos de fracturas faciales (Figura 5). En caso de no contar con equipos de imágenes tridimensionales existen otras técnicas que pueden ser útiles. Es importante evaluar la indicación de imagenología sobre todo en pacientes pediátricos ${ }^{30,31}$.

Si no se tiene un equipo de tomografía, se puede complementar el estudio con otras técnicas radiográficas, por ejemplo: las fracturas mandibulares se pueden evaluar mediante una ortopantomografía (radiografía panorámica) y una radiografía de Towne invertida (posteroanterior). En caso de no contar con ortopantomógrafo puede ser tomada una radiografía lateral oblicua bilateral.

Las fracturas de arco cigomático pueden ser evaluadas con una radiografía submentovertex (cabeza en hiperextensión con plano de Frankfurt perpendicular al piso). En caso de fractura nasal se puede utilizar una radiografía de huesos propios nasales.

\section{Conclusión}

Es de gran importancia que el médico urgenciólogo tenga las competencias para resolver este tipo de cuadros, sobre todo si se desenvuelve en una zona rural, donde el acceso a un servicio especializado es más difícil.

El manejo del trauma y la atención de situaciones con amenaza inmediata a la vida deben ser tratadas con prioridad, sin embargo, no se deben dejar de lado el manejo de lesiones que por un mal diagnóstico, tratamiento o derivación pueden llevar a pérdidas de función, alteraciones del desarrollo o deformidades secundarias de las estructuras faciales. En base a esto es que realizamos y presentamos un algoritmo para el manejo de urgencias en cirugía maxilofacial por no especialistas (Figura 6).

Se recalca la importancia de un examen físico acabado y apoyo por imágenes necesarios de acuerdo a cada caso y contexto. 


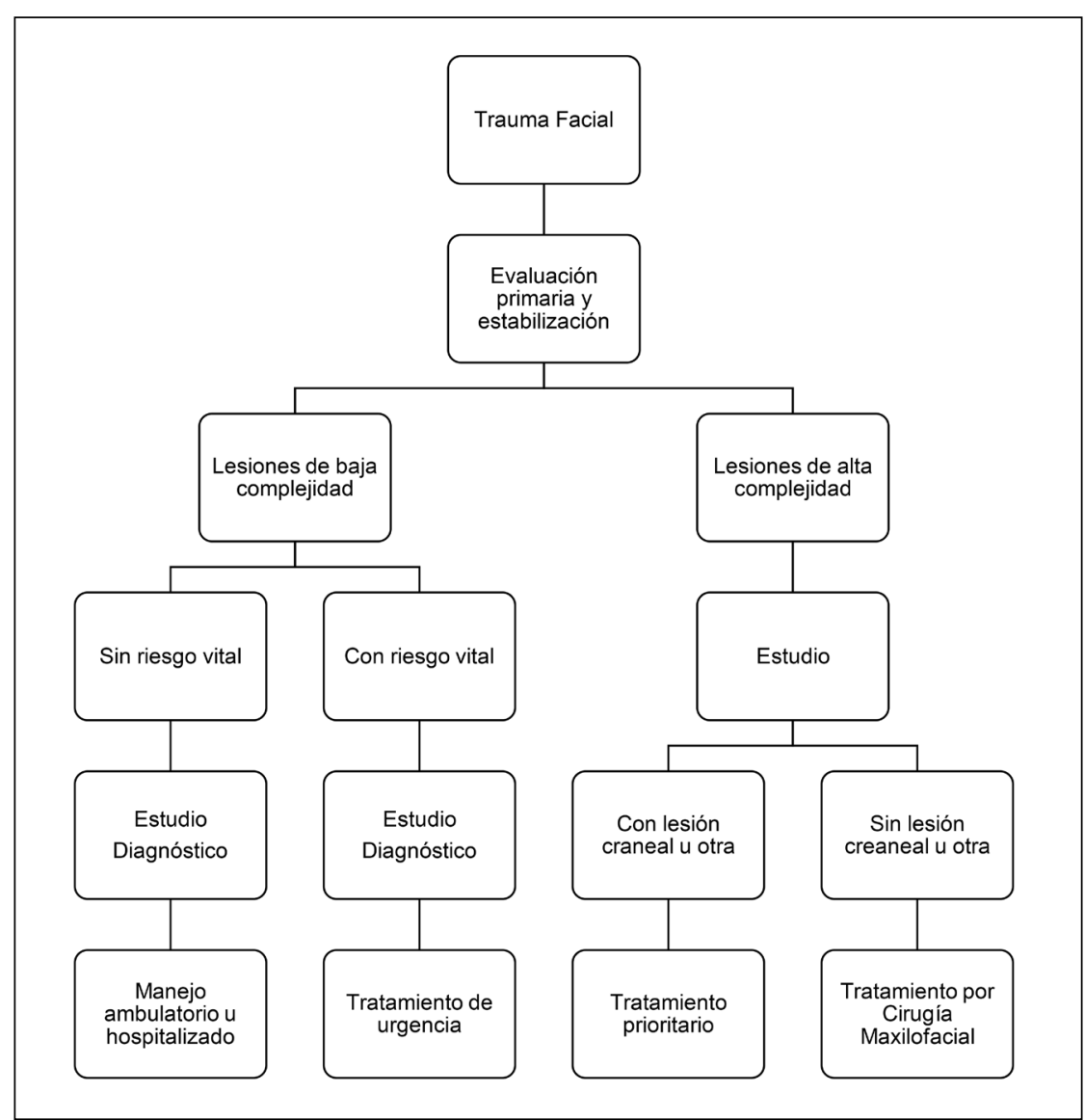

Figura 6. Algoritmo manejo de urgencia en trauma maxilofacial. *Fracturas nasal simple, fractura dentoalveolar, fractura malar. ${ }^{* *}$ Fracturas Le Fort, naso-orbito-etmoidales, nasal compleja, panfacial.

\section{Referencias}

1. Peden M, Sharma G. The injury chart book: a graphical overview of the global burden of injuries. World Health Organization: Geneva, 2002.

2. Kotecha S. A four year retrospective study of 1,062 patients presenting with maxillofacial emergencies at a specialist paediatric hospital. The British journal of oral \& maxillofacial surgery 2008; 46 (4): 293-6.

3. Lee J, Cho B, and Park W. A 4-year retrospective study of facial fractures on Jeju, Korea. J Craniomaxillofac Surg 2010; 38 (3): 192-6.

4. Gassner R, Tuli T, Hächl O, Rudisch A, Ulmer H. Cranio-maxillofacial trauma: a 10 year review of 9,543 cases with 21,067 injuries. J Craniomaxillofac Surg 2003; 31 (1): 51-61.

5. Brasileiro B, Passeri L. Epidemiological analysis of maxilofacial fractures in Brazil: a 5-year prospective study.
Oral Surg Oral Med Oral Pathol Oral Radiol Endod 2006; 102 (1): 28-34.

6. Medina M, Bobadilla P, Zaror R, Olate S. Maxillofacial fractures in chilean subjects. Int J Morphol 2006; 24 (3): 423-8.

7. Down K, Boot D, Gorman D. Maxillofacial and associated injuries in severely traumatized patients: implications of a regional survey. Int J Oral Maxillofac Surg 1995; 24 (6): 409-12.

8. DeAngelis A, Barrowman RA, Harrod R, Nastri AL. Review article: Maxillofacial emergencies: Maxillofacial trauma. Emerg Med Australas 2014; 26 (6): 530-7.

9. Trunkey D. Trauma. Accidental and intentional injuries account for more years of life lost in the U.S. than cancer and heart disease. Among the prescribed remedies are improved preventive efforts, speedier surgery and further research. Sci Am 1983; 249 (2): 28-35.

10. Demetriades D, Kimbrell B, Salim A, Velmahos G, Rhee 
$\mathrm{P}$, Preston C, et al. Trauma deaths in a mature urban trauma system: is "trimodal" distribution a valid concept? J Am Coll Surg 2005; 201 (3): 343-8.

11. Andersson L, Kahnberg K, Pogrel M. Oral and Maxillofacial Surgery. 2012: Wiley.

12. Sasser S, Hunt RC, Faul M, Sugerman D, Pearson WS, Dulski T, et al. Guidelines for field triage of injured patients: recommendations of the National Expert Panel on Field Triage, 2011. MMWR Recomm Rep 2012; 61 (RR-1): 1-20.

13. Perry M. Advanced Trauma Life Support (ATLS) and facial trauma: can one size fit all? Part 1: dilemmas in the management of the multiply injured patient with coexisting facial injuries. Int J Oral Maxillofac Surg 2008; 37 (3): 209-14.

14. Miloro M, Ghali G, Larsen P, Waite P. Peterson's principles of oral and maxillofacial surgery. 2nd ed. London, 2004.

15. Perry M, Morris C. Advanced trauma life support (ATLS) and facial trauma: can one size fit all? Part 2: ATLS, maxillofacial injuries and airway management dilemmas. Int J Oral Maxillofac Surg 2008; 37 (4): 30920.

16. Flavell E, Stacey M, Hall J. The clinical management of airway obstruction. Current Anaesthesia and Critical Care 20 (3): 102-12.

17. Veeravagu A, Joseph R, Jiang B, Lober RM, Ludwig C, Torres R, et al. Traumatic epistaxis: Skull base defects, intracranial complications and neurosurgical considerations. Int J Surg Case Rep 2013; 4 (8): 656-61.

18. Genu P, de Oliveira D, Vasconcellos R, Nogueira R, Vasconcelos B. Inadvertent intracranial placement of a nasogastric tube in a patient with severe craniofacial trauma: a case report. J Oral Maxillofac Surg 2004; 62 (11): 1435-8.

19. LynhamA, Hirst J, Cosson J, Chapman P, McEniery P. Emergency department management of maxillofacial trauma. Emerg Med Australas 2004; 16 (1): 7-12.

20. Lalani $Z$, Bonanthaya $\mathrm{K}$. Cervical spine injury in maxillofacial trauma. Br J Oral Maxillofac Surg 1997; 35 (4): 243-5.

21. Mukherjee S, Abhinav K, Revington P. A review of cer- vical spine injury associated with maxillofacial trauma at a UK tertiary referral centre. Ann R Coll Surg Engl 2015; 97 (1): 66-72.

22. Tuckett J, Lynham A, Lee G, Perry M, Harrington U. Maxillofacial trauma in the emergency department: a review. Surgeon 2014; 12 (2): 106-14.

23. Hoffman J, Wolfson A, Todd K, Mower W. Selective cervical spine radiography in blunt trauma: methodology of the National Emergency X-Radiography Utilization Study (NEXUS). Ann Emerg Med 1998; 32 (4): 461-9.

24. Stiell I, Clement C, McKnight R, Brison R, Schull M, Rowe B. The Canadian C-spine rule versus the NEXUS low-risk criteria in patients with trauma. N Engl J Med 2003; 349 (26): 2510-8.

25. Hoffman J, Mower W, Wolfson A, Todd K, Zucker M. Validity of a set of clinical criteria to rule out injury to the cervical spine in patients with blunt trauma. National Emergency X-Radiography Utilization Study Group. N Engl J Med 2000; 343 (2): 94-9.

26. Perry M, O’Hare J, Porter G. Advanced Trauma Life Support (ATLS) and facial trauma: can one size fit all? Part 3: Hypovolaemia and facial injuries in the multiply injured patient. Int J Oral Maxillofac Surg 2008; 37 (5): 405-14.

27. MINSAL. Guía Clínica Traumatismo Cráneo Encefálico Moderado o Grave. Santiago, Chile. 2013.

28. Ellis E, Scott K. Assessment of patients with facial fractures. Emerg Med Clin North Am 2000; 18 (3): 411-48, vi.

29. Perry M, Moutray T. Advanced Trauma Life Support (ATLS) and facial trauma: can one size fit all? Part 4: 'can the patient see?' Timely diagnosis, dilemmas and pitfalls in the multiply injured, poorly responsive/unresponsive patient. Int J Oral Maxillofac Surg 2008; 37 (6): 505-14.

30. Feng S, Law M, Huang B, Ng S, Li Z, Meng Q, et al. Radiation dose and cancer risk from pediatric CT examinations on 64-slice CT: a phantom study. Eur J Radiol 2010; 76 (2): e19-23.

31. Mehta N, Butala P, Bernstein M. The imaging of maxillofacial trauma and its pertinence to surgical intervention. Radiol Clin North Am 2012; 50 (1): 43-57. 